\title{
Seleção de atributos em avaliações sensoriais descritivas
}

\author{
Karina Rossini ${ }^{a^{*}}$, Michel José Anzanello ${ }^{\mathrm{b}}$, \\ Flavio Sanson Fogliatto ${ }^{c}$ \\ a*karinarossini@hotmail.com, UFRGS, Brasil \\ banzanello@producao.ufrgs.br, UFRGS, Brasil \\ cffogliatto@producao.ufrgs.br, UFRGS, Brasil
}

\begin{abstract}
Resumo
A seleção dos atributos a serem avaliados em uma análise sensorial é fundamental no planejamento de painéis sensoriais. 0 processo de seleção visa reduzir a lista de atributos a serem apresentados aos julgadores, evitando assim fadiga aos membros do painel, porém mantendo atributos significativos na caracterização das amostras avaliadas. Este artigo apresenta um método para seleção de atributos em painéis sensoriais baseados em avaliações descritivas das amostras, tais como os métodos QDA (Quantitative Descriptive Analysis) e Spectrum. 0 método proposto utiliza Análise de Componentes Principais para identificação dos atributos mais relevantes e então aplica Análise Discriminante para classificação das amostras em formulações distintas. 0 método é aplicado em um estudo de caso em que cubos de carne com molho são caracterizados em painel sensorial utilizando o método QDA. 0 método proposto reduz significativamente o número de atributos a serem avaliados e conduz à satisfatória acurácia de classificação das amostras em formulações.
\end{abstract}

Palavras-chave

Seleção de atributos. Análise sensorial. Análise multivariada.

\section{Introdução}

Na indústria de alimentos, a análise sensorial é elemento chave para identificar as expectativas dos consumidores (LEDAUPHIN; POMMERET; QANNARI, 2008). A análise sensorial compreende um conjunto de técnicas para medir precisamente atributos sensoriais de produtos a partir de respostas humanas. Tais técnicas utilizam princípios oriundos da ciência de alimentos, fisiologia, psicologia e estatística, fornecendo respostas objetivas para as propriedades de alimentos, conforme percebidas pelos cinco sentidos (PIGGOTT; SIMPSON; WILLIAMS, 1998).

Informações obtidas através de avaliações sensoriais podem ser utilizadas por empresas como suporte técnico para pesquisa, industrialização, marketing e controle de qualidade dos itens produzidos, qualificando decisões técnicas e administrativas. Na perspectiva do consumidor, a avaliação sensorial em produtos industriais assegura que cheguem ao mercado com as características desejadas (DUTCOSKY, 1996; LAWLESS; HEYMANN, 1998).

A avaliação sensorial é realizada de acordo com diferentes testes que dependem da sua finalidade (ANZALDÚA-MORALES, 1994). Limitações comuns nesses testes referem-se ao número de amostras, número de julgadores ou quantidade de atributos a serem analisados. No que tange a esse último aspecto, Sahmer e Qannari (2008) apontam que a seleção de atributos tem sido foco de investigações em diversas áreas industriais. Em particular, no perfil sensorial descritivo, estratégias de seleção podem ser utilizadas para reduzir o conjunto de atributos, selecionando aqueles relevantes e não redundantes. Tal seleção reduz o tempo de execução, a fadiga imposta aos membros do painel, e os custos da avaliação. 
Um atributo significativo em uma investigação sensorial é aquele cuja avaliação apresenta diferenças sistemáticas e significativas entre as amostras investigadas, tal que seja possível relacionar o nível do atributo (através de seu valor medido) com características das amostras (por exemplo, a presença ou ausência de um ingrediente). Atributos que apresentam tal comportamento poderiam, por exemplo, ser utilizados como variáveis de resposta em modelos de regressão, os quais permitiriam a otimização do produto investigado no painel sensorial. Neste artigo, propõe-se um método para identificação de tais atributos usando dados obtidos em uma análise sensorial preliminar usando métodos descritivos.

0 método proposto utiliza ferramentas de análise multivariada de dados, conciliando a Análise de Componentes Principais (ACP) e a Análise Discriminante (AD) como meio para redução do número de atributos (variáveis) na avaliação sensorial. Os pesos gerados pela ACP são transformados em índices de importância dos atributos. Esses índices são vinculados à $A D$, a qual classifica as amostras em diferentes classes de formulação, e uma medida de desempenho da classificação (geralmente acurácia) é calculada. Na sequência, o atributo apontado como menos relevante pelo índice de importância é eliminado e uma nova classificação é realizada, acompanhada por uma nova medição de acurácia. Esse procedimento iterativo é finalizado ao atingir-se um número limite de atributos remanescentes, e o subconjunto de atributos responsável pela maior acurácia é retido. Por fim, um procedimento de enumeração permite refinar o subconjunto anteriormente gerado pela inclusão dos atributos eliminados imediatamente antes do ponto de maior acurácia ter sido atingido. Tal procedimento assegura a obtenção de um subconjunto com elevado poder de discriminação das amostras.

$\mathrm{Na} A C P$, as variáveis são reescritas como combinações lineares, as quais são denominadas componentes principais. Cada componente descreve uma porção da variabilidade presente nas variáveis originais, e sua interpretação é geralmente baseada na magnitude dos pesos associados às variáveis. Apesar do número de componentes principais resultantes de uma ACP sobre um conjunto de variáveis ser igual ao número de variáveis analisadas, usualmente é possível obter uma boa representação dos dados utilizando um número reduzido de componentes (RENCHER, 1995). A AD, por sua vez, é uma técnica de classificação e discriminação de amostras que permite alocar novas observações a grupos pré-determinados. Na utilização da $A D$, um grupo de observações cujos membros já estão identificados é utilizado para estimar pesos (ou parâmetros) de uma função discriminante conforme alguns critérios, tal como a minimização de erros de classificação (SUEYOSHI; GOTO, 2009).
0 presente artigo traz uma importante contribuição para a área da Sensometria: o desenvolvimento de procedimentos de seleção de atributos, usuais em estudos de classificação, aplicados a problemas de análise sensorial. Os métodos QDA e Spectrum, de ampla utilização prática, estão baseados na caracterização plena das amostras através de um grande número de atributos, muitos dos quais não contribuem na diferenciação das amostras investigadas. 0 método aqui proposto permite identificar tais atributos utilizando dados de uma análise preliminar, eliminando-os da coleta posterior de dados, envolvendo um maior número de julgadores. Otimiza-se, assim, a coleta de dados sensoriais, usualmente de alto custo no desenvolvimento de produtos industriais.

0 presente trabalho é composto de cinco seções. Além da introdução, é apresentado um referencial teórico sobre seleção de variáveis, seguido pela descrição do método proposto e, posteriormente, apresenta-se o estudo aplicado, finalizando com as conclusões.

\section{Referencial teórico}

As seções seguintes apresentam abordagens para a identificação dos atributos mais relevantes em análises sensoriais, além de descrever os fundamentos das técnicas multivariadas utilizadas no método proposto (Análise de Componentes Principais e Análise Discriminante).

\subsection{Seleção de atributos em análises sensoriais}

Técnicas analíticas multivariadas têm sido amplamente utilizadas em diversas áreas do conhecimento, motivadas por variadas aplicações. $\mathrm{Na}$ indústria de alimentos, em particular no tratamento de dados sensoriais, ressalta-se seu envolvimento para fins de (i) previsão das propriedades sensoriais e caracterização de produtos; (ii) seleção e identificação de segmentos de consumidores; e (iii) seleção de atributos em análises sensoriais descritivas.

Com vistas à previsão das propriedades sensoriais e caracterização de produtos, Johansen et al. (2008), em pesquisa com iogurtes desnatados e cream cheese, utilizaram a Regressão por Mínimos Quadrados Parciais (PLSR, ou partial least squares regression) para relacionar os resultados da análise sensorial descritiva a imagens digitais das superfícies das amostras. Karoui et al. (2006) fizeram uso de PLSR e Análise de Correlação Canônica (CCA, ou canonical correlation analysis) para comparar os resultados da análise sensorial com os obtidos através do Infravermelho 
Próximo (NIR, ou near infra red) em queijos, ao passo que Esteban-Díez, González-Sáiz e Pizarro (2004) utilizaram estas mesmas técnicas para investigar as relações entre determinados atributos sensoriais do café expresso e dados espectrais de amostras de café torrado. Por fim, Kreutzmann et al. (2008) utilizaram uma abordagem multibloco (LS-ParPLS) para a caracterização de cenouras.

Uma sistemática para identificação de segmentos de consumidores foi proposta por Sahmer, Vigneau e Qannari (2006) através da combinação de análise de cluster e algoritmo hierárquico em estudos com café. Heenan et al. (2009) avaliaram três segmentos de consumidores quanto à percepção acerca de pães frescos através da Análise de Componentes Principais (APC) e análise de cluster, utilizando também PLSR para investigar a relação entre as percepções dos consumidores para cada segmento. Carbonell et al. (2008) investigaram a segmentação de consumidores através do uso de um coeficiente de correlação entre consumidores e atributos sensoriais. Naquele trabalho, a segmentação, por meio da análise de clusters, fundamentou-se na semelhança entre os coeficientes de correlação dos consumidores.

A seleção de atributos em análises sensoriais descritivas, tema do presente artigo, é abordado por outro grupo de pesquisadores. Dijksterhuis, Frøst e Byrne (2002) propuseram uma aplicação em análise sensorial para o método estatístico proposto em Krzanowski (1995): o estudo objetivou reduzir um grande grupo de variáveis determinantes da percepção da gordura no leite em subgrupos menores, utilizando ACP. Os autores utilizaram uma rotação procrustes nos subgrupos, sendo o subgrupo detentor da menor perda procrustes selecionado. Já Sahmer e Qannari (2008) compararam seis métodos distintos para identificação de um subconjunto de atributos sensoriais relevantes em dados descritivos oriundos de perfis sensoriais. Dois dos métodos testados baseiam-se em ACP, dois utilizam a análise generalizada de procrustes (GPA, ou generalized procrustes analysis) e dois envolvem análise de clusters. A análise visou reduzir o número total de atributos apresentados ao painel e preservar a estrutura multivariada dos dados. Os métodos utilizando análise de clusters obtiveram melhores resultados em conjuntos de dados reais e simulados. Porém, apesar de proporcionar a identificação dos atributos relevantes, o método desenvolvido pelos autores não dispensa a coleta de dados sobre atributos considerados não relevantes.

Westada et al. (2003) apresentam uma metodologia para identificação de atributos significantes em modelos multivariados, baseada na aplicação de ACP em dados de análise sensorial descritiva e de consumidores. 0 objetivo é interpretar fatores latentes que abrangem características como sabor, odor, aparência e textura em sorvetes e queijos, identificando produtos semelhantes ou diferentes, bem como os atributos que os diferenciam. 0 método foi baseado nas estimativas de incerteza provenientes da técnica de cross-validation/Jack-knifing, sendo o teste $t$ de student utilizado para calcular a significância de cada variável para cada componente.

Carbonell, lzquierdo e Carbonell (2007) utilizaram a GPA para selecionar os atributos na avaliação de sucos natural e processado de tangerina. Peron (2000) identificou as diferenças entre variedades de batatas através de trinta atributos avaliados por 14 julgadores. Para tanto, selecionaram-se atributos de acordo com sua capacidade discriminativa utilizando ANOVA (Análise de Variância) e ACP. Por fim, o autor utilizou uma GPA normalizada para calcular o índice confiabilidade de cada julgador. Outro método para seleção de atributos discriminantes, desta vez aplicado a diferentes tipos de queijos, foi proposto por Granitto et al. (2007). Os autores compararam o método random forests (RFs) com análise discriminante linear (LDA, ou Linear Discriminant Analysis) e discriminante por mínimos quadrados parciais (DPLS, ou Discriminant Partial Least Squares). A função discriminante derivada do RFs atribui pesos de importância para os atributos, sendo esses pesos responsáveis pela seleção de um número reduzido de atributos que garanta um satisfatório poder discriminatório da função.

\subsection{Análise de Componentes Principais e Análise Discriminante}

A Análise de Componentes Principais (ACP) tem o objetivo de reduzir a dimensionalidade de um conjunto de dados sem perda significativa de informações. Os componentes principais são combinações lineares dos dados originais. Através da ACP é possível substituir os dados originais por um número reduzido de componentes principais independentes entre si (JACKSON, 1980, 1981).

Considere um conjunto de dados composto de realizações de $p$ atributos sensoriais. É possível extrair desse conjunto de dados $p$ componentes principais, sendo cada um uma combinação linear distinta dos $p$ atributos originais. Cada componente principal (CP) captura uma direção de variabilidade do conjunto de dados originais. As direções capturadas por cada componente principal são ortogonais entre si (ou seja, garantindo a independência entre CPs).

A determinação dos componentes principais pode utilizar as informações na matriz de covariâncias ou de correlações associadas aos $p$ atributos originais. 
A matriz de covariâncias é recomendada quando a escala dos atributos analisados pode fornecer informações relevantes sobre a estrutura dos dados (RENCHER, 1995). No caso de atributos descritos por escalas oriundas de questionário ou painéis, geralmente contidas no mesmo intervalo de valores, as análises feitas através da matriz de covariâncias e de correlação geram resultados semelhantes, a menos que haja efeito significativo de localização no uso da escala pelos diferentes julgadores. Nesse caso, recomenda-se a remoção de tais efeitos através de pré-tratamento dos dados (por exemplo, usando o método proposto em Ledauphin, Hanafi e Qannari, 2006) ou o uso da matriz de correlações na ACP.

Seja $\Sigma$ a matriz de covariâncias, de dimensão $(p \times p)$, associada à matriz de dados observados para os atributos $\mathrm{X}=\left[\mathrm{x}_{1}, \mathrm{x}_{2}, \ldots, \mathrm{x}_{p}\right]$. A dimensão de $\mathrm{X}$ é $(n \times p)$, ou seja, dispõem-se de $n$ observações de cada atributo. 0 vetor $x^{t}$ denota uma linha qualquer de $X$, isto é, $x_{1}=\left(x_{i 1}, x_{i 2}, \ldots, x_{i n}\right)$ sendo $i=1,2, \ldots, p$ e $\mathrm{n}$ o tamanho da amostra. Os $p$ autovalores de $\Sigma$ são designados por $\lambda, i=1, \ldots, p$, e os $p$ autovetores normalizados designados por $\mathrm{w}_{,} i=1, \ldots, p$, com elementos dados por $\left(w_{i 1}, \ldots, w_{i p}\right)$. Associado a cada autovalor $\lambda_{i}$ existe um autovetor $\mathrm{w}_{i}$ Assim, os pares $\left(\lambda_{1}, \mathrm{w}_{1}\right),\left(\lambda_{2}, \mathrm{w}_{2}\right), \ldots,\left(\lambda_{p}, \mathrm{w}_{p}\right)$ correspondem aos autovalores e autovetores de $\Sigma$, com autovalores arranjados tal que $\lambda_{1} \geq \lambda_{2} \geq \ldots . \geq \lambda_{p}$. $0 i$-ésimo componente principal pode ser obtido pela expressão (SEBER, 1984):

$$
Y_{i}=\chi^{t} w_{i}=w_{i 1} X_{1}+w_{i 2} X_{2}+\ldots+w_{i p} X_{p}
$$

0 número de componentes principais a ser retido $(M)$ pode ser definido com base no percentual de variância explicado pelos componentes. Para tanto, somam-se os autovalores (arranjados do maior ao menor) até atingir-se um valor desejado de variância (RENCHER, 1995). 0 percentual ideal da variância explicada pelos PCs depende da natureza do problema analisado.

$\mathrm{Na}$ Equação 1, os elementos do autovetor $\mathrm{w}_{i}$ funcionam como pesos de importância dos atributos $X_{1}, \ldots, X_{p}$ na composição do $i$-ésimo $\mathrm{CP}$, e são denominados cargas do componente. Em CPs obtidos a partir de atributos padronizados, a magnitude do peso associado ao atributo descreve importância relativa deste na composição dos CPs.

No restante desta seção, apresentam-se os fundamentos da Análise Discriminante (AD).

A AD é uma técnica de classificação de observações em classes distintas (JOBSON, 1996). Diferentemente das técnicas tradicionais de clusterização, a AD possibilita a classificação de novos objetos nas classes já existentes sem que seja necessário um rearranjo de classes (MINGOTI, 2005). Além do propósito de classificação, a AD também pode ser utilizada para obter um melhor entendimento das relações entre as observações e os grupos a que pertencem.

A técnica é operacionalizada através da construção de uma relação entre uma variável dependente não contínua $(Z)$ e um conjunto de variáveis contínuas $(x ’ s)$. Essa relação é expressa através de uma função discriminante consistindo em uma combinação das variáveis contínuas (HAIR et al., 2006; ZANINE et al., 2008). Duas funções tradicionalmente geradas são as do tipo linear aplicada quando as matrizes de covariâncias são similares entre as classes analisadas e exemplificadas na Equação 2 ou do tipo quadrática, quando as matrizes de covariâncias entre as classes são distintas. Outras funções podem ser encontradas em Hair et al. (2006).

$$
Z=b_{0}+b_{1} x_{1}+\ldots+b_{p} x_{p}
$$

onde $b_{0}$ é uma constante, $b_{i}$ são os coeficientes de ponderação associados às variáveis independentes, e $Z$ é o escore discriminante obtido para cada objeto na análise.

Por constituir-se em uma técnica supervisionada (DUDA; HART; STORK, 2001), os coeficientes $b_{i}$ da $A D$ são estimados com base em um banco de dados em que a classe de cada observação é conhecida a priori. Os coeficientes são determinados com base na média e na variância-covariância das variáveis pertencentes a cada classe, maximizando-se a diferença padronizada entre os escores médios provenientes de classes distintas. A classificação de observações futuras é feita através da determinação do seu escore $Z$, e posterior comparação com limites estabelecidos. Mais detalhes acerca da geração da função discriminante e obtenção dos coeficientes podem ser encontrados em Hair et al. (2006).

\section{Método proposto}

0 método aqui proposto é baseado em uma sequência de etapas que utilizam, majoritariamente, duas técnicas de análise multivariada de dados: ACP e AD. Os pesos dos componentes principais da ACP geram subsídios para a identificação dos atributos que apresentam maior variabilidade, servindo com base para a geração de um índice de importância dos atributos. A AD, por sua vez, cumpre a função de categorizar as observações (amostras) nas classes de formulação com base nos atributos. Um processo iterativo de classificação via AD e eliminação dos atributos menos relevantes é balizado pelo índice de importância gerado para os atributos. 
A aplicação do método pressupõe uma pré-coleta de dados sensoriais utilizando métodos descritivos, tais como o QDA (Quantitative Descriptive Analysis; ver STONE et al., 1974; STONE; SIDEL, 1992) e o método Spectrum (ver MEILGAARD; CARR; CIVILLE, 1999). A principal característica desse tipo de método é a mensuração da intensidade de atributos em amostras utilizando escalas contínuas. Na pré-coleta, utiliza-se um número reduzido de julgadores e repetições. De posse dos dados da pré-coleta, aplicam-se as etapas detalhadas no restante desta seção.

0 método fundamenta-se na seguinte lógica. Deseja-se identificar o conjunto de atributos que maximiza a diferença entre as formulações, aqui tratadas como classes, avaliadas no painel sensorial. Quanto maior a diferença entre classes, maior a chance da obtenção de modelos de regressão significativos, associando atributos a ingredientes testados em diferentes níveis nas formulações. Tais modelos viabilizam a otimização da formulação do produto analisado usando procedimentos de otimização de experimentos multirresposta (ver FOGLIATTO; ALBIN, 2001, entre outros). Desta forma, atributos a serem retidos são aqueles responsáveis por parcelas importantes da variabilidade observada entre classes. 0 resultado de um painel sensorial fornecido por um julgador é uma observação; nela, uma amostra, correspondendo a uma formulação, é analisada com relação a $p$ atributos. Conhece-se, a priori, a qual classe pertence cada observação obtida na análise sensorial do produto, já que estão vinculadas às formulações testadas; o objetivo aqui é identificar quais atributos melhor caracterizam essa alocação de observações em classes.

\subsection{Aplicação da ACP no banco de dados e geração do índice de importância dos atributos}

A ACP é aplicada na matriz de correlação dos atributos e tem por objetivo gerar-lhes pesos de importância. Tais pesos, dados pelo autovetor $\mathrm{w}_{i}$ associado ao $i$-ésimo componente principal, além de identificar atributos que apresentam maior variabilidade, são utilizados na geração de um índice de importância para cada atributo (designado por $I A$ ).

0 índice de importância do atributo $i$ é estimado com base na magnitude dos pesos gerados pela ACP. Este índice é composto pela soma dos valores absolutos dos pesos sobre os $M$ componentes retidos, conforme apresentado na Equação 3.

$$
I A_{i}=\sum_{j=1}^{R}\left|w_{i j}\right| i=1, \ldots, p
$$

Atributos com valores de $I A$ elevados na Equação 3 são considerados mais relevantes na análise, uma vez que apresentam maior variância associada. Segundo Duda, Hart e Stork (2001) e Anzanello, Albin e Chaovalitwongse (2009), atributos com elevada variância são desejados em procedimentos de classificação/discriminação, visto que possibilitam separações mais precisas entre os grupos.

\subsection{Eliminação dos atributos irrelevantes para classificação das amostras em classes de formulação}

Neste passo, almeja-se identificar os atributos mais relevantes para classificação das amostras em classes de formulação. Para tanto, adota-se uma sistemática de eliminação dos atributos menos relevantes no formato backward (ANZANELLO, 2009), em que os atributos são sistematicamente eliminados do banco de dados após cada classificação.

0 procedimento de classificação proposto aplica $\mathrm{AD}$ sobre os $p$ atributos. 0 desempenho da classificação das amostras (observações) nas classes pré-definidas (formulações) é estimado através da acurácia. A acurácia é definida como a razão entre o número de classificações corretas e o número total de classificações efetuadas. É importante enfatizar que o procedimento de classificação não se apoiou nas tradicionais porções de treino (construção do modelo) e de teste (validação em novas observações) em decorrência do limitado número de repetições dentro de cada combinação de formulação e julgador. Logo, a acurácia de classificação foi estimada com base nas mesmas observações utilizadas para construção da função discriminante.

Na sequência, o atributo com o menor valor de $I A$ é eliminado do banco de dados, e os $p$ - 1 atributos remanescentes são utilizados em uma nova classificação das amostras. A acurácia de classificação é novamente calculada. Esse procedimento iterativo é mantido até atingir-se um número desejado de atributos remanescentes, sendo que dois atributos são o limite inferior recomendado.

Um gráfico associando acurácia e número de atributos retidos é gerado para monitorar o processo de eliminação. Esse gráfico auxilia na definição de um subconjunto apropriado de atributos, o qual é analisado em detalhes na etapa seguinte do método.

\subsection{Análise do gráfico de acurácia e definição do melhor subconjunto de atributos}

Essa etapa é composta por duas subetapas: (i) definição de um ponto de corte no gráfico de acurácia e seleção de um subconjunto de 
atributos, $S A$; e (ii) enumeração das possíveis combinações dos atributos em $S A$ (incluindo um número limitado de atributos recentemente eliminados, $A E$ ), e seleção do subconjunto de atributos responsável pela máxima acurácia de classificação.

A definição de um ponto de corte no gráfico de acurácia é feita de forma subjetiva, e visa definir um número mínimo de atributos a ser considerado no procedimento de enumeração. Recomenda-se escolher um ponto que concilie um nível de acurácia satisfatório e um número reduzido de atributos retidos. Essa condição é verificada próxima a pontos em que a acurácia apresenta considerável redução com a eliminação de um atributo, conforme exemplo na Figura 1.

Na sequência, recomenda-se incluir alguns dos atributos recentemente eliminados (ou seja, pertencentes ao subconjunto $A E$ ) ao subconjunto $S A$ (conforme Figura 1). Esse procedimento visa assegurar que atributos de importância intermediária sejam inseridos no procedimento de enumeração, podendo eventualmente gerar acurácias satisfatórias ao serem combinados aos atributos em $S A$. A não inclusão de elementos de $A E$ em $S A$ não compromete a eficiência do método, mas pode conduzir a acurácias inferiores. 0 número de atributos de $A E$ é definido subjetivamente (recomenda-se de 3 a 5 atributos), sendo escolhidos os atributos com valor de $I A$ imediatamente superior ao $I A$ do último atributo incluído no subconjunto $S A$.

A subetapa (ii) consiste na enumeração total de combinações $\left\lfloor\left(\begin{array}{l}m \\ b\end{array}\right)\right.$, onde $m$ refere-se aos atributos em $S A+A E$ e $b$ denota o possível número de atributos a serem mantidos, variando de $\{S A\}$ a $\{S A+A E\}$, onde $\{\phi\}$ denota o número de elementos em $\phi]$. Cada combinação tem sua acurácia de classificação avaliada, sendo que a combinação responsável pela máxima acurácia indica o melhor subconjunto de atributos.

Por fim, enfatiza-se que uma análise simplificada pode ser conduzida através da seleção direta dos atributos responsáveis pelo ponto de corte no gráfico de acurácia (subconjunto $S A$ ), eliminando-se assim a necessidade de enumeração.

\section{Estudo de caso}

Nesta seção, aplica-se o método proposto para seleção de atributos utilizando dados obtidos em uma avaliação sensorial realizada utilizando o método Spectrum de análise sensorial descritiva. Trata-se de um dos mais rigorosos métodos para treinamento e coleta de informações sensoriais de julgadores, desenvolvido por Gail Civille e apresentado em Meilgaard, Carr e Civille (1999).

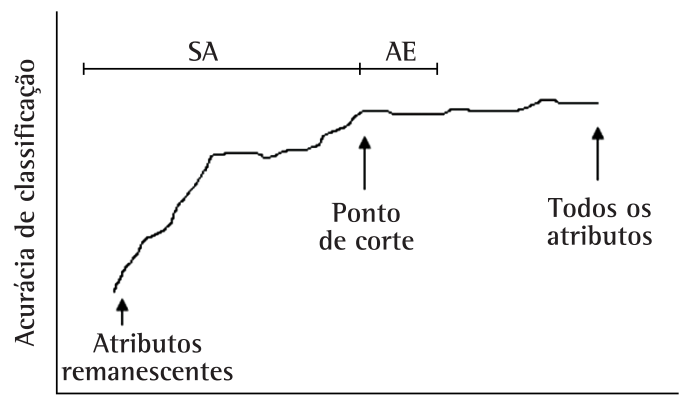

Número de atributos retidos

Figura 1. Ponto de corte e atributos retidos na análise.

Julgadores candidatos a integrar o painel foram selecionados em duas etapas. A primeira etapa consistiu de uma entrevista telefônica, com o objetivo de identificar a disponibilidade do candidato e sua familiaridade com testes sensoriais. Candidatos considerados aptos foram convidados a realizar uma bateria completa de testes, nos quais atributos relacionados a sabor, textura e aparência foram investigados. De aproximadamente 25 candidatos, 8 foram selecionados ao término da fase preliminar de treinamento ( 3 meses). A segunda fase consistiu de duas sessões de treinamento semanais, de duração aproximada de 1,5 horas, durante mais 3 meses, ao cabo dos quais os julgadores foram considerados treinados.

Os painéis sensoriais buscavam a caracterização de uma ração militar constituída de cubos de carne ao molho embalados em pouches termoestáveis. Os oito julgadores analisaram 26 atributos de 8 formulações do produto em questão. Cada avaliação foi realizada em quadriplicata. Os painéis sensoriais foram realizados em 1994, no Nabisco Food Center da Rutgers University (EUA), como parte de um projeto de pesquisa para o exército norte-americano. Os atributos avaliados estão agrupados por similaridade, conforme apresentado na Tabela 1.

ACP foi aplicada na matriz de correlação dos atributos padronizados. A matriz de correlação e a variância dos atributos são apresentadas no Apêndice. Os 24 atributos (os atributos 3 e 4 foram descartados da análise por apresentarem dados incompletos) foram tratados como variáveis e as amostras como observações ( 8 formulações $\times 8$ julgadores $\times 4$ repetições). Foram retidos 4 componentes principais, respondendo por $68 \%$ da variância total. A inclusão de componentes adicionais não eleva a acurácia da classificação, de acordo com os testes realizados. Os pesos dos componentes foram manipulados através da Equação 3, gerando os índices de importância $I A$ para os 24 atributos, conforme apresentado na Tabela 2 (observe que os atributos são apresentados em ordem decrescente de importância). 
Tabela 1. Atributos sensoriais.

\begin{tabular}{|c|c|c|}
\hline Identificação (ID) & Quesito & Atributo sensorial \\
\hline 1 & \multirow{5}{*}{ Aparência } & Proporção de molho na carne \\
\hline 2 & & Espessura visual do molho \\
\hline 3 & & Cor do molho (dados não avaliados) \\
\hline 4 & & Cor dos cubos da carne (dados não avaliados) \\
\hline 5 & & Uniformidade de tamanho e forma da carne \\
\hline 6 & \multirow{8}{*}{ Sabor } & Aroma de carne cozida \\
\hline 7 & & Aroma de caldo de carne \\
\hline 8 & & Aroma de carne crua \\
\hline 9 & & Aroma de proteína vegetal hidrolisada \\
\hline 10 & & Carne com sangue coagulado \\
\hline 11 & & Espessura \\
\hline 12 & & Aroma de carne queimada \\
\hline 13 & & Aroma de gordura \\
\hline 14 & Sabores básicos & Sal \\
\hline 15 & \multirow{2}{*}{ Sensações } & Sensação metálica \\
\hline 16 & & Sensação de calor \\
\hline 17 & \multirow{5}{*}{ Textura } & Viscosidade do molho \\
\hline 18 & & Elasticidade da carne \\
\hline 19 & & Densidade da carne \\
\hline 20 & & Coesividade inicial da carne \\
\hline 21 & & Firmeza da carne \\
\hline 22 & \multirow{4}{*}{ Características de mastigação } & Maciez da carne \\
\hline 23 & & Fibrosidade da carne \\
\hline 24 & & Estratificação da carne \\
\hline 25 & & Umidade da carne \\
\hline 26 & Características residuais & Película oleosa \\
\hline
\end{tabular}

Tabela 2. Pesos absolutos dos 4 componentes principais (CP) retidos e índice de importância (IA) em ordem decrescente de importância.

\begin{tabular}{|c|c|c|c|c|c|}
\hline \multirow{2}{*}{$\begin{array}{l}\text { ID do } \\
\text { atributo }\end{array}$} & \multicolumn{4}{|c|}{ Pesos absolutos } & \multirow{2}{*}{$I A$} \\
\hline & CP1 & CP2 & CP3 & $\mathrm{CP} 4$ & \\
\hline 10 & 0,157 & 0,229 & 0,191 & 0,305 & 0,881 \\
\hline 2 & 0,196 & 0,057 & 0,091 & 0,509 & 0,853 \\
\hline 16 & 0,003 & 0,282 & 0,314 & 0,236 & 0,835 \\
\hline 17 & 0,121 & 0,100 & 0,098 & 0,494 & 0,813 \\
\hline 21 & 0,092 & 0,327 & 0,364 & 0,026 & 0,810 \\
\hline 18 & 0,243 & 0,223 & 0,274 & 0,069 & 0,808 \\
\hline 22 & 0,112 & 0,223 & 0,421 & 0,049 & 0,804 \\
\hline 15 & 0,023 & 0,425 & 0,321 & 0,025 & 0,794 \\
\hline 19 & 0,002 & 0,393 & 0,152 & 0,206 & 0,753 \\
\hline 9 & 0,405 & 0,152 & 0,026 & 0,136 & 0,719 \\
\hline 26 & 0,211 & 0,311 & 0,183 & 0,009 & 0,713 \\
\hline 6 & 0,296 & 0,094 & 0,132 & 0,146 & 0,668 \\
\hline 20 & 0,422 & 0,039 & 0,158 & 0,034 & 0,652 \\
\hline 1 & 0,180 & 0,211 & 0,044 & 0,214 & 0,648 \\
\hline 7 & 0,045 & 0,206 & 0,215 & 0,144 & 0,609 \\
\hline 8 & 0,260 & 0,036 & 0,140 & 0,147 & 0,582 \\
\hline 25 & 0,150 & 0,116 & 0,030 & 0,265 & 0,562 \\
\hline 23 & 0,445 & 0,044 & 0,004 & 0,066 & 0,559 \\
\hline 5 & 0,037 & 0,094 & 0,285 & 0,142 & 0,557 \\
\hline 14 & 0,168 & 0,032 & 0,174 & 0,142 & 0,516 \\
\hline 11 & 0,081 & 0,160 & 0,051 & 0,213 & 0,505 \\
\hline 12 & 0,012 & 0,074 & 0,273 & 0,037 & 0,396 \\
\hline 13 & 0,098 & 0,122 & 0,054 & 0,065 & 0,339 \\
\hline 24 & 0,006 & 0,156 & 0,023 & 0,045 & 0,231 \\
\hline
\end{tabular}

Na sequência, iniciou-se o processo iterativo de classificação e eliminação dos atributos (descrito na seção 3.2), gerando-se o gráfico de acurácia da Figura 2. A avaliação subjetiva deste gráfico mostra que o perfil de acurácia permanece estável à medida que os primeiros 11 atributos são removidos, e então existe uma queda considerável na acurácia (ponto destacado no perfil e definido como ponto de corte). Tal ponto corresponde a 14 atributos retidos.

A Tabela 3 apresenta os valores de acurácia após cada eliminação de atributo (bem como a identificação do atributo eliminado). A primeira linha numérica desta tabela apresenta 0,723 como a acurácia de classificação obtida quando 24 atributos são considerados; na linha seguinte, o atributo 24 é eliminado, e a acurácia de classificação utilizando os 23 atributos remanescentes cai para 0,711 . 0 ponto de corte selecionado refere-se ao subconjunto formado por 14 atributos, os quais conduzem a uma acurácia de 0,676 . Tal subconjunto (definido como subconjunto $S A)$, que contém representantes de todos os quesitos pesquisados, é formado pelos seguintes atributos, em ordem decrescente de importância: 10, 2, 16, 17, 21, 18, 22, 15, 19, 9, 26, 6, 20 e 1.0 subconjunto $A E$ é constituído pelos 5 atributos removidos antes que o ponto de corte fosse atingido (atributos 7, 8, 25, 23 e 5), na Tabela 2. 


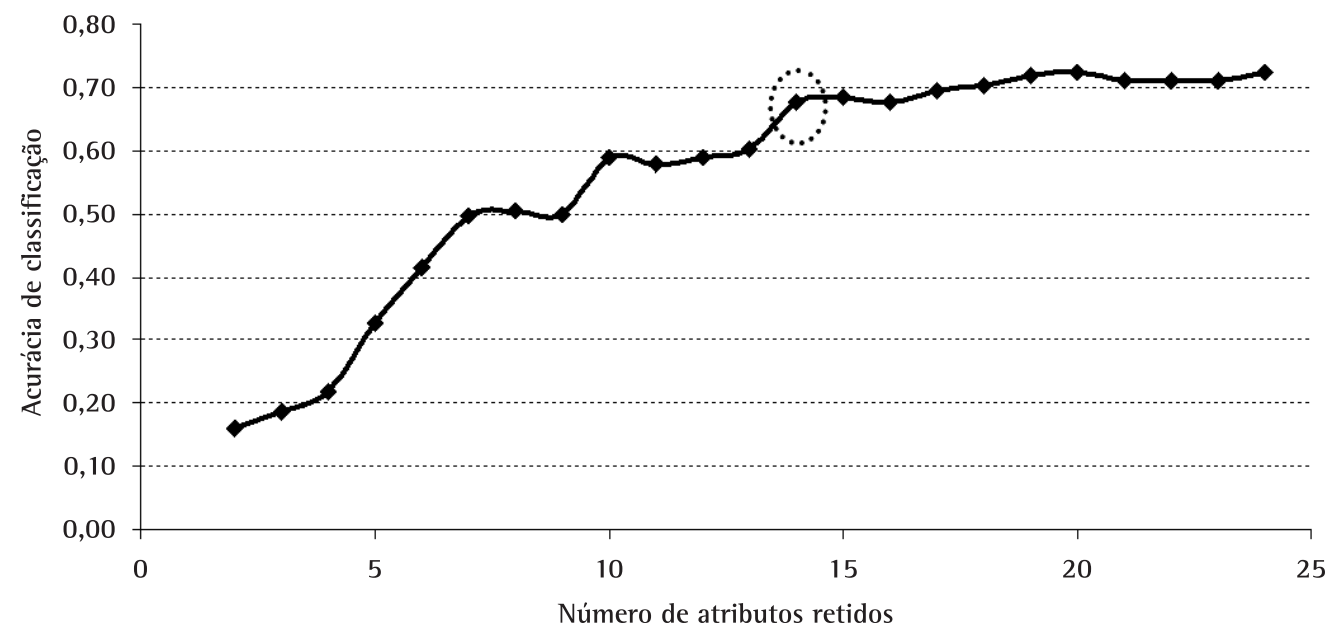

Figura 2. Perfil de acurácia gerado pela remoção de atributos.

A enumeração das possíveis combinações incluiu os atributos $S A+A E$ acima listados. 0 número de atributos a serem retidos variou de 14 a 19; o primeiro valor é arbitrado como o número mínimo de atributos a serem mantidos com base no ponto de corte, enquanto que 19 reflete a inclusão dos 5 atributos oriundos de $A E$. Por exemplo, a primeira enumeração testou a acurácia de todos os possíveis subconjuntos de 14 atributos formados a partir dos 19 atributos candidatos $(S A+A E)$, resultando em um valor de 0,714. A Tabela 4 traz a maior acurácia gerada pela retenção de diferentes números de atributos. Este procedimento confirma que 16 atributos devem ser retidos, o que assegura uma acurácia de 0,723 , valor idêntico ao obtido quando todos os atributos são utilizados. Os atributos selecionados são: 1, 2, 5, 6, 7, 8, 9, $10,16,17,19,20,21,23,25$ e 26 . Observe que, dentre os atributos selecionados, figuram todos os atributos de aparência, bem como a maioria dos atributos de sabor.

Por fim, a acurácia do método proposto (0,723 com 16 atributos) foi comparada à máxima acurácia possível, obtida através da enumeração total de combinações geradas pelos 24 atributos (i.e., $\left(\begin{array}{c}24 \\ 1\end{array}\right)+\ldots+\left(\begin{array}{c}24 \\ 24\end{array}\right)$ ). 0 máximo valor de acurácia possível é 0,742, obtido com 19 atributos (os 19 atributos responsáveis pela acurácia de 0,742 são diferentes dos 19 atributos selecionados pelo método, acima apresentados). Vale enfatizar que a enumeração total das possibilidades demandou 32 horas de processamento em PC $2.4 \mathrm{GHz}$, ao passo que a enumeração decorrente do método proposto demandou 10 minutos de análise.
Tabela 3. Identificação do atributo eliminado.

\begin{tabular}{ccc}
\hline Acurácia & Número de atributos retidos & $\begin{array}{c}\text { ID do atributo } \\
\text { eliminado }\end{array}$ \\
\hline 0,723 & 24 & - \\
0,711 & 23 & 24 \\
0,711 & 22 & 13 \\
0,711 & 21 & 12 \\
0,723 & 20 & 11 \\
0,719 & 19 & 14 \\
0,703 & 18 & 5 \\
0,695 & 17 & 23 \\
0,676 & 16 & 25 \\
0,684 & 15 & 8 \\
0,676 & 14 & 7 \\
0,602 & 13 & 1 \\
0,589 & 12 & 20 \\
0,578 & 11 & 6 \\
0,589 & 10 & 26 \\
0,500 & 9 & 9 \\
0,503 & 8 & 19 \\
0,496 & 7 & 15 \\
0,414 & 6 & 22 \\
0,328 & 5 & 18 \\
0,218 & 4 & 21 \\
0,187 & 3 & 17 \\
0,160 & 2 & 16 \\
\hline Atributos posicionados além do limite de & 2 \\
& eliminação & 10 \\
\hline
\end{tabular}

Tabela 4. Variação da acurácia com o número de atributos retidos no procedimento de enumeração.

\begin{tabular}{cc}
\hline Acurácia & Número de atributos retidos \\
\hline 0,714 & 14 \\
0,719 & 15 \\
0,723 & 16 \\
0,723 & 17 \\
0,719 & 18 \\
0,719 & 19 \\
\hline
\end{tabular}




\section{Conclusões}

Em painéis de avaliação sensorial, a existência de um número elevado de atributos conduz a procedimentos longos e caros, além de impor fadiga aos membros do painel. De tal forma, a seleção dos atributos mais relevantes constitui-se em uma importante etapa no planejamento desses painéis.

Este estudo apresentou um método para seleção de atributos na avaliação sensorial de alimentos. 0 método concilia duas metodologias de análise multivariada: análise de componentes principais (ACP) e análise discriminante (AD). Os pesos gerados pela ACP dão origem a um índice de importância dos atributos. $A A D$ é então utilizada para classificação das amostras em diferentes classes, denotando formulações distintas. Um procedimento iterativo é desencadeado através da classificação das amostras e subsequente eliminação dos atributos menos relevantes, de acordo com a ordem definida pelos índices de importância. A acurácia de classificação é avaliada após cada eliminação de atributo, indicando um subconjunto de potenciais atributos a serem utilizados. Por fim, um procedimento de enumeração identifica o melhor subconjunto de atributos a serem considerados em painéis sensoriais.

0 método proposto permitiu reduzir o número de atributos em um painel de análise sensorial de cubos de carne ao molho de 24 para 16, mantendo-se os mesmos níveis de acurácia. Por fim, verificou-se que o resultado gerado pela metodologia proposta é similar à máxima acurácia possivel, a qual é obtida de forma exaustiva por enumeração sobre a totalidade de atributos.

\section{Referências}

ANZALDÚA-MORALES, A. A. La evaluación sensorial de los alimentos en la teoria e la práctica. Zaragoza: Editorial Acribia S.A., 1994.

ANZANELLO, M. J. Seleção de variáveis com vistas à classificação de bateladas de produção em duas classes. Gestão \& Produção, v. 16, p. 526-533, 2009.

ANZANEllO, M. J.; AlBIN, S.; CHAOVALITWONGSE, W. A., Selecting the best variables for classifying production batches into two quality levels. Chemometrics and Intelligent Laboratory Systems, v. 97, p. 111-117, 2009. http://dx.doi.org/10.1016/j.chemolab.2009.03.004

CARBONELL, L.; IZQUIERDO, L.; CARBONELL, 1. Sensory analysis of Spanish mandarin juices. Selection of attributes and panel performance. Food Quality and Preference, v. 18, p. 329-341, 2007. http://dx.doi. org/10.1016/j.foodqual.2006.02.008

CARBONELL, L. et al. Segmentation of food consumers according to their correlations with sensory attributes projected on preference spaces. Food Quality and Preference, v. 19, p. 71-78, 2008. http://dx.doi. org/10.1016/j.foodqual.2007.06.006
DIJKSTERHUIS, G.; FRøST, M. B.; BYRNE, D. V. Selection of a subset of variables: minimisation of Procrustes loss between a subset and the full set. Food Quality and Preference, v. 13, p. 89-97, 2002. http://dx.doi. org/10.1016/S0950-3293(01)00065-9

DUDA, R.; HART, P.; STORK, D. Pattern Classification. 2. ed. New York: Wiley-Interscience, 2001.

DUTCOSKY, S. D. Análise Sensorial de Alimentos. Curitiba: Champagnat, 1996.

ESTEBAN-DíEZ, 1.; GONZÁLEZ-SÁlZ, J. M.; PIZARRO, C. Prediction of sensory properties of espresso from roasted coffee samples by near-infrared spectroscopy. Analytica Chimica Acta, v. 525, p. 171-182, 2004. http://dx.doi. org/10.1016/j.aca.2004.08.057

FOGLIATTO, F. S.; ALBIN, S. L. A hierarchical method for evaluating products with quantitative and sensory characteristics. llE Transactions, v. 33, p. 1081-1092, 2001. http://dx.doi.org/10.1080/07408170108936898

GRANITTO, P. M. et al. Modern data mining tools in descriptive sensory analysis: A case study with a Random forest approach. Food Quality and Preference, v. 18, p. 681-689, 2007. http://dx.doi.org/10.1016/j. foodqual.2006.11.001

HAIR, J. F. et al. Análise multivariada de dados. New York: Bookman, 2006.

HEENAN, S. P. et al. Characterization of fresh bread flavor: Relationships between sensory characteristics and volatile composition. Food Chemistry, v. 116, p. 249-257, 2009. http://dx.doi.org/10.1016/j.foodchem.2009.02.042

LAWLESS, H. T.; HEYMANN, H. Sensory Evaluation of Food: Principles and Practices. New York: Chapman \& Hall, 1998.

JACKSON, J. E. Principal Component and factor Analysis: Part 1 - Principal Components. Journal of Quality Technology, v. 12, p. 201-213, 1980.

JACKSON, J. E. Principal Component and factor Analysis: Part 11 - Additional Topics Related to Principal Components. Journal of Quality Technology, v. 13, p. 46-58, 1981.

JOBSON, J. D. Applied multivariate data analysis. New York: Springer-Verlag, 1996.

JOHANSEN, S. M. B. et al. Prediction of sensory properties of low-fat yoghurt and cream cheese from surface images. Food Quality and Preference, v. 19, p. 232-246, 2008. http://dx.doi.org/10.1016/j.foodqual.2007.03.006

KAROUI, R. et al. Prediction of sensory attributes of European Emmental cheese using near-infrared spectroscopy: A feasibility study. Food Chemistry, v. 101, p. 1121-1129, 2006. http://dx.doi.org/10.1016/j. foodchem.2006.03.012

KRZANOWSKl, W. Selection of variables, and assessment of their performance, in mixed-variable discriminant analysis. Computational Statistics and Data Analysis, v. 19, p. 419-431, 1995. http://dx.doi.org/10.1016/01679473(94)00011-7

KREUTZMANN, S. et al. Prediction of sensory quality in raw carrots (Daucus carota L.) using multi-block LS-ParPLS. Food Quality and Preference, v. 19, p. 609-617, 2008. http://dx.doi.org/10.1016/j.foodqual.2008.03.007

LEDAUPHIN, S.; HANAFI, M.; QANNARI, E. M. Assessment of the agreement among the subjects in fixed vocabulary profiling. Food Quality and Preference, 
v. 17, p. 277-280, 2006. http://dx.doi.org/10.1016/j. foodqual.2005.03.017

LEDAUPHIN, S.; POMMERET, D.; QANNARI, M. Application of hidden Markov model to products shelf lives. Food Quality and Preference, v.19, p.156-161, 2008. http:// dx.doi.org/10.1016/j.foodqual.2007.04.006

MEILGAARD, M. C.; CARR, B. T.; CIVILlE, G. V. Sensory Evaluation Techniques. 4. ed. Boca Ratón: CRC Press, 1999. http://dx.doi.org/10.1201/9781439832271

MINGOTI, S. A. Análise de dados através de métodos de estatística multivariada: uma abordagem aplicada. Belo Horizonte: UFMG, 2005.

PERON, L. Statistical analysis of sensory profiling data: data reductionand generalised Procrustes analysis. Food Quality and Preference, v.11, p.155-157, 2000. http:// dx.doi.org/10.1016/S0950-3293(99)00070-1

PIGGOTT, J. R.; SIMPSON, S. J.; WILLIAMS, S. A. R. Sensory analysis. International Journal of Food Science and Technology, v. 33, p. 7-18, 1998. http://dx.doi. $\operatorname{org} / 10.1046 / j .1365-2621.1998 .00154 . x$

RENCHER, A. C. Methods of Multivariate Analysis. New York: Wiley, 1995.

SAHMER, K.; VIGNEAU, E.; QANNARI, E. M. A cluster approach to analyze preference data: choice of the number of clusters. Food Quality and Preference, v.17, p.257-265, 2006. http://dx.doi.org/10.1016/j. foodqual.2005.03.007
SAHMER, K.; QANNARI, E. M. Procedures for the selection of a subset of attributes in sensory profiling. Food Quality and Preference, v. 19, p. 141-145, 2008. http://dx.doi. org/10.1016/j.foodqual.2007.03.007

SEBER, G. A. F. Multivariate observations. New York: Wiley, 1984. http://dx.doi.org/10.1002/9780470316641

STONE, H.; SIDEL, J. Sensory Evaluation Practices. 2. ed. San Diego: Academic Press, 1992.

STONE, H. et al. Sensory Evaluation by Quantitative Descriptive Analysis. Food Technology, v. 28, n. 1, p. 2434, 1974.

SUEYOSHI, T.; GOTO, M. Methodological comparison between DEA (data envelopment analysis) and DEADA (discriminant analysis) from the perspective of bankruptcy assessment. European Journal of Operational Research, v. 199, n. 2, p. 561-575, 2009. http://dx.doi. org/10.1016/j.ejor.2008.11.030

WESTADA, F. et al. Variable selection in PCA in sensory descriptive and consumer data. Food Quality and Preference, v. 14, p. 463-472, 2003. http://dx.doi. org/10.1016/S0950-3293(03)00015-6

ZANINE, A. M. et al. Evaluation of the grass-tanzania ("Panicum maximum") using multivariate analyses. Revista Brasileira de Saúde e Produção Animal, v. 9, p. 179-189, 2008.

\title{
Attribute selection in descriptive sensory analysis
}

\begin{abstract}
The selection of attributes from a group of candidates to be assessed through sensory analysis is an important step when planning sensory panels. When selecting attributes, it is desirable to reduce the list of those to be presented to panelists in order to avoid fatigue, however keeping the attributes that are relevant to the sensory characterization of samples. This paper presents a multivariate method for attribute selection in descriptive sensory panels, such as those used in the QDA (Quantitative Descriptive Analysis) and Spectrum protocols. The proposed method is implemented using Principal Component Analysis and Descriptive Analysis, and it is evaluated in a case study where beef cubes in stew, used as combat ration by the American Army, are characterized in sensory panels through the Spectrum method. The method significantly reduced the number of attributes to be considered in sensory panels, while yielding satisfactory accuracy in the classification of samples.
\end{abstract}

Keywords

Attribute selection. Sensory evaluation. Multivariate analysis. 


\begin{tabular}{|c|c|c|c|c|c|c|c|c|c|c|c|c|c|c|c|c|c|c|c|c|c|c|c|c|}
\hline 㱐 & في & $\underset{-}{\stackrel{\Delta}{0}}$ & 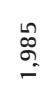 & : & 员 & $\begin{array}{l}\text { م⿱ } \\
0 \\
0 \\
0\end{array}$ & $\begin{array}{l}0.0 \\
\text { on } \\
0\end{array}$ & $\begin{array}{l}\text { जे } \\
\text { के }\end{array}$ & $\begin{array}{c}\bar{m} \\
\text { c. }\end{array}$ & $\begin{array}{l}\overline{0} \\
\& \\
0\end{array}$ & $\begin{array}{l}\text { 怘 } \\
\text { 足 }\end{array}$ & $\begin{array}{l}0 \\
\vdots \\
=\end{array}$ & $\begin{array}{l}\text { \$े } \\
\text { s. }\end{array}$ & $\stackrel{m}{\underline{m}}$ & $\hat{\tilde{g}}$ & $\begin{array}{l}\tilde{N} \\
\tilde{N}\end{array}$ & ठे & & & & & & & 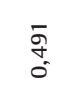 \\
\hline$\stackrel{\sim}{\sim}$ & $\frac{n}{0}$ & $\stackrel{\circ}{\circ}$ & 8 & $\frac{2}{0}$ & $\begin{array}{l}\overline{0} \\
0\end{array}$ & $\overline{\bar{i}}$ & $\tilde{o}$ & $\begin{array}{l}\text { O̊ } \\
\text { i. }\end{array}$ & $\stackrel{n}{0}_{0}^{0}$ & $\frac{0}{i}$ & $\begin{array}{l}\text { ָे } \\
\text { cै }\end{array}$ & i. & $\frac{n}{0}$ & $\frac{\partial}{\partial}$ & $\begin{array}{l}\infty \\
\mathbb{s}^{\circ}\end{array}$ & $\begin{array}{l}8 \\
i \\
i\end{array}$ & & & & & & & & \\
\hline ผิ & 8 & $\begin{array}{l}\text { a } \\
0 \\
i\end{array}$ & $\begin{array}{l}\stackrel{n}{0} \\
0\end{array}$ & $\bar{y}_{0}$ & $\stackrel{0}{\circ}$ & to & s. & $\begin{array}{l}\text { Ĩ } \\
\text { ô }\end{array}$ & $\begin{array}{l}n \\
0 \\
0\end{array}$ & $\begin{array}{l}\overline{0} \\
0 \\
i\end{array}$ & $\frac{0}{0}$ & $\begin{array}{l}0 \\
0 \\
0 \\
0\end{array}$ & to & 8 & $\stackrel{n}{0}$ & $\begin{array}{l}\infty \\
0 \\
0 \\
0\end{array}$ & & $\bar{\approx}$ & & n & & & & \\
\hline$\stackrel{\Delta}{\sim}$ & त̃ & $\underset{0}{\tilde{0}}$ & $\begin{array}{l}0 \\
0 \\
i\end{array}$ & f̃ & $\begin{array}{l}\stackrel{n}{0} \\
0 \\
0\end{array}$ & $\begin{array}{l}0 \\
\text { rn } \\
\text { î }\end{array}$ & $\begin{array}{l}\bar{a} \\
\bar{i}\end{array}$ & $\overline{0}$ & $\begin{array}{l}\text { of } \\
0.1\end{array}$ & ô. & $\stackrel{\simeq}{\circ}$ & $\bar{i}$ & $\begin{array}{l}\bar{i} \\
\bar{i}\end{array}$ & $0^{\circ}$ & $\stackrel{\circ}{0}$ & $\begin{array}{l}0 \\
+ \\
0 \\
0\end{array}$ & & & & $\begin{array}{l}\stackrel{2}{0} \\
0\end{array}$ & & & & \\
\hline ָ & $\stackrel{\tilde{c}}{0}$ & స̃. & $\stackrel{n}{0}$ & f & $\frac{n}{0}$ & $\begin{array}{c}m \\
\hat{1} \\
\hat{p}_{0}\end{array}$ & $\bar{o}_{0}$ & ஸે & $T$ & ¿ै. & $\begin{array}{l}\text { N̂ } \\
\text { Oे }\end{array}$ & & 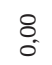 & ¿ & $\frac{m}{0}$ & $\begin{array}{l}\text { f } \\
\text { pi }\end{array}$ & is & ర & & : & & & & \\
\hline$\approx$ & $\tilde{o}_{0}$ & $\begin{array}{l}\stackrel{n}{0} \\
0 \\
i\end{array}$ & $\begin{array}{l}\tilde{N} \\
\delta\end{array}$ & 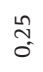 & $\begin{array}{l}\hat{0} \\
0 \\
i\end{array}$ & $\frac{6}{i}$ & 8 & $\begin{array}{l}\bar{o} \\
i \\
i\end{array}$ & $\tilde{0}$ & $\begin{array}{l}0 \\
0 \\
0 \\
i\end{array}$ & $\overbrace{0}^{0}$ & $\begin{array}{l}\tilde{n} \\
\underline{0} \\
i\end{array}$ & $\frac{0}{i}$ & & $\stackrel{0}{-}$ & స̃. & $\tilde{m}_{0}$ & $\bar{F}$ & & 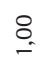 & ๙ & & & \\
\hline $\bar{\sim}$ & $\therefore$ & $\frac{n}{i}$ & ח̃ & $\begin{array}{l}\text { to } \\
\text { i }\end{array}$ & $\overline{0}$ & $\begin{array}{l}\tilde{O} \\
\stackrel{0}{0}\end{array}$ & $\stackrel{\infty}{\circ}$ & $\begin{array}{l}\text { o. } \\
\text { i. }\end{array}$ & $\bar{\sigma}$ & $\begin{array}{l}\hat{0} \\
0 \\
i\end{array}$ & $\begin{array}{l}\hat{0} \\
0 \\
i\end{array}$ & & ${ }_{0}^{0}$ & $\tilde{0}$ & $\overline{\bar{i}}$ & 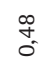 & $\begin{array}{l}+ \\
0 \\
0\end{array}$ & ำ & & s & & & & \\
\hline ำ & $\stackrel{\stackrel{n}{0}}{0}$ & $\begin{array}{l}\stackrel{2}{0} \\
\text { - }\end{array}$ & $\overline{0}$ & $\underset{0}{f}$ & ô. & $\begin{array}{l}\stackrel{n}{n} \\
\hat{\rho}\end{array}$ & 若 & ్ㅗㅇ & & $\begin{array}{l}\infty \\
0 \\
0 \\
i\end{array}$ & $\begin{array}{l}\stackrel{2}{0} \\
\text { ó }\end{array}$ & $\begin{array}{l}\hat{\lambda} \\
\text { ît }\end{array}$ & $\begin{array}{l}8 \\
0 \\
i\end{array}$ & & & $\begin{array}{l}\stackrel{2}{1} \\
\text { ó }\end{array}$ & $\stackrel{\text { L }}{0}$ & & & $\bar{\sigma}$ & 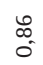 & & & \\
\hline I & $\frac{\pi}{0}$ & $\frac{\circ}{0}$ & $\begin{array}{l}8 \\
0 \\
0\end{array}$ & $\tilde{o}_{0}^{0}$ & $\sigma$ & $\frac{\circ}{i}$ & 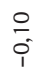 & $\stackrel{n}{0}$ & $\frac{\pi}{0}$ & $\frac{N}{i}$ & $\frac{t}{0}$ & ஜิ & $\begin{array}{l}\stackrel{1}{0} \\
\text { O- }\end{array}$ & $m$ & $\hat{0}_{0}$ & ֻุ & 8 & & 车 & $m$ & $\begin{array}{l}0 \\
0 \\
0 \\
0\end{array}$ & & & \\
\hline$\stackrel{\infty}{\circ}$ & $=$ & $\begin{array}{l}\text { ñ } \\
\text { ô }\end{array}$ & $\frac{0}{0}$ & $\bar{o}_{0}$ & $\frac{m}{i}$ & $\begin{array}{l}\tilde{O} \\
\text { o }\end{array}$ & $\begin{array}{l}\text { to } \\
\text { i. }\end{array}$ & $\begin{array}{l}\text { స్ } \\
\text { i. }\end{array}$ & $\bar{o}_{0}$ & $\begin{array}{l}0 \\
0 \\
i\end{array}$ & $\begin{array}{l}\infty \\
0 \\
i \\
i\end{array}$ & O্ & $\bar{o}_{0}$ & 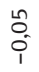 & $\begin{array}{l}\text { त̃ } \\
\text { î }\end{array}$ & $\stackrel{8}{-}$ & ֻั & & & הู & ? & & & \\
\hline$=$ & $\frac{\infty}{0}$ & $\hat{\tilde{n}}$ & $\tilde{0}_{0}$ & ò & 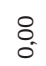 & $\frac{\infty}{p^{-}}$ & $\hat{0}$ & $\frac{0}{0}$ & is & $\begin{array}{l}\infty \\
\stackrel{0}{0} \\
0\end{array}$ & $\frac{0}{0}$ & $\delta_{0}$ & 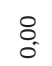 & 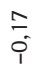 & $\stackrel{8}{-}$ & $\begin{array}{l}\text { N. } \\
\text { î }\end{array}$ & 2 & & & p & & & & \\
\hline$\underline{\varrho}$ & $\begin{array}{c}\bar{c} \\
\text { ó }\end{array}$ & $\begin{array}{l}\bar{z} \\
\bar{i}\end{array}$ & $\overline{0}$ & $\begin{array}{l} \pm \\
\vdots \\
0\end{array}$ & t & $\frac{t}{0}$ & $\begin{array}{l}0 \\
0 \\
0\end{array}$ & $\begin{array}{l}\infty \\
: \\
0\end{array}$ & s. & $\begin{array}{l}\text { L్ } \\
\text { ó }\end{array}$ & స్ & $\overline{\overline{0}^{\circ}}$ & q & & $\frac{\bar{c}}{i}$ & $\begin{array}{l}\text { مo } \\
0 \\
0 \\
1\end{array}$ & $\frac{f}{5}$ & & & $\therefore$ & $\overbrace{0}^{2}$ & & & \\
\hline$\underline{\Omega}$ & 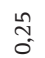 & 8 & $\underbrace{t}_{0}$ & $\bar{a}$ & $\frac{m}{0}$ & $\overline{0}$ & $\stackrel{2}{2}$ & F & है & $\begin{array}{c}\tilde{N}_{0} \\
\sigma_{0}\end{array}$ & $\begin{array}{l}0 \\
+ \\
0 \\
0\end{array}$ & $\frac{1}{5}$ & $\underset{-}{8}$ & & 8 & $\begin{array}{l}\overline{0}_{0} \\
0\end{array}$ & $\begin{array}{l}\text { : } \\
\text { s. }\end{array}$ & & & & & & & \\
\hline \pm & $\frac{\Delta}{0}$ & $\tilde{o}_{0}^{0}$ & $\begin{array}{l}n \\
0 \\
i\end{array}$ & $\stackrel{\Upsilon}{े}_{i}$ & 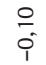 & त̃ & $\begin{array}{l}\tilde{0} \\
0 \\
0\end{array}$ & $\begin{array}{l}\stackrel{0}{1} \\
0\end{array}$ & & 8 & $\begin{array}{l}0_{0}^{\circ} \\
0 \\
\end{array}$ & 8 & $\simeq$ & $=$ & $\stackrel{\tilde{O}}{0}$ & $\begin{array}{l}\text { t. } \\
0 \\
0\end{array}$ & $\tilde{o}_{0}^{0}$ & & & G & & & & \\
\hline m & $\stackrel{\tilde{O}}{0}$ & $\frac{m}{0}$ & $\hat{o}_{0}$ & लె & $\stackrel{\text { å }}{0}$ & $\overline{0}$ & o & $\tilde{m}_{0}$ & & $\frac{\theta}{0}$ & 8 & 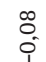 & $\begin{array}{l}\text { fo } \\
0\end{array}$ & & $\frac{\circ}{0}$ & 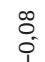 & & & & 6 & & & & \\
\hline$N$ & ô & $\frac{0}{0}$ & $\stackrel{\simeq}{\circ}$ & $\stackrel{2}{0}$ & $=$ & $\stackrel{n}{=}$ & $\hat{0}$ & $\frac{t}{0}$ & & $\stackrel{8}{-}$ & $\frac{t}{0}$ & $:$ & त̃ & $\begin{array}{l}\text { : } \\
\text { s. }\end{array}$ & $\begin{array}{l}\infty \\
: \\
0\end{array}$ & $\tilde{O}_{0}$ & & & & s. & & & & \\
\hline$=$ & $\Xi$ & $\stackrel{n}{\circ}$ & $\approx$ & $\breve{c}$ & व & $\bar{N}$ & to & o. & $\stackrel{8}{-}$ & $\infty$ & $\begin{array}{l}8 \\
0 \\
0\end{array}$ & a & $\begin{array}{l}\stackrel{\sim}{N} \\
\text { o }\end{array}$ & & & 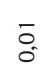 & & & & s. & & & & \\
\hline 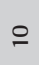 & : & $\begin{array}{l}\stackrel{\infty}{\sim} \\
\stackrel{0}{0}\end{array}$ & $\begin{array}{l}0 \\
0 \\
0\end{array}$ & $\stackrel{\infty}{0}$ & $\begin{array}{l}0 \\
0 \\
i\end{array}$ & 3 & 7 & 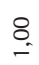 & & \pm & $\tilde{m}_{0}$ & 0 & $\mp$ & & & o̊ & & & & $\begin{array}{l}\overline{0} \\
0 \\
i\end{array}$ & & & & \\
\hline$\sigma$ & $\approx$ & $\begin{array}{l}t \\
0 \\
0\end{array}$ & $\begin{array}{l}t \\
0 \\
0\end{array}$ & t & $\begin{array}{l}n \\
0 \\
0\end{array}$ & $\begin{array}{l}0 \\
0 \\
0\end{array}$ & \& & 1 & to & ô. & ô. & $\begin{array}{l}0 \\
0 \\
0\end{array}$ & \begin{tabular}{l}
0 \\
\multirow{1}{1}{} \\
0
\end{tabular} & & $\hat{o}_{0}$ & $\begin{array}{l}1 \\
0 \\
0\end{array}$ & & $0^{\circ}$ & & bis & & & & \\
\hline$\infty$ & $\bar{\sim}$ & $\stackrel{\sim}{\sim}$ & $\overline{0}$ & $\tilde{\sigma}$ & $\stackrel{0}{0}$ & 8 & oี & $\stackrel{\simeq}{\circ}$ & & مـ & $=$ & సิ & $=$ & & & 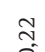 & & & & & & & & \\
\hline 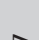 & $\bar{a}$ & I & $=$ & ธี & 0 & 8 & $m$ & $\approx$ & & & $\infty$ & & & & 0 & & & & & & & & & \\
\hline & & i & & & & & S & & & & p & & & & & & & & & & & & & \\
\hline ๑ & 0 & $\begin{array}{l}\infty \\
0 \\
0\end{array}$ & 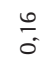 & $\stackrel{8}{-}$ & 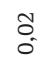 & $\tilde{m}$ & ${ }_{0}^{+}$ & $\infty$ & & $\begin{array}{l}0 \\
0\end{array}$ & $\tilde{c}_{0}^{2}$ & & $\overline{0}$ & & 1 & $\begin{array}{l}\overline{0} \\
0\end{array}$ & $\begin{array}{l}\tilde{0} \\
0 \\
0\end{array}$ & & & $\hat{N}$ & f & & & \\
\hline\llcorner & \&̊ & 8 & $\stackrel{8}{\circ}$ & $\frac{0}{0}$ & 0 & $\begin{array}{l}\overline{0} \\
0\end{array}$ & $\begin{array}{l}0 \\
0 \\
0\end{array}$ & 9 & & $\frac{\sim}{i}$ & o. & 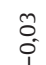 & $\begin{array}{l}t \\
0 \\
0\end{array}$ & 5 & 0 & $\frac{6}{0}$ & $b^{\circ}$ & & 2 & 5 & 5 & & & \\
\hline$\sim$ & $\stackrel{\infty}{0}$ & $\underset{-}{8}$ & $\begin{array}{l}8 \\
0 \\
0\end{array}$ & $\stackrel{\infty}{:}$ & d & $\begin{array}{l}\hat{N} \\
\hat{i}\end{array}$ & to & 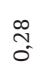 & & $\frac{0}{0}$ & $\frac{m}{0}$ & $\begin{array}{l}\Re \\
0 \\
0\end{array}$ & 8 & ít & $0^{\circ}$ & $\begin{array}{l}\text { ñ } \\
\underline{i}\end{array}$ & $0^{\circ}$ & 0 & & i & 5 & & & \\
\hline- & व & $\begin{array}{l}\infty \\
0 \\
0 \\
i\end{array}$ & ฮี & $\stackrel{\text { İ }}{\text { Jै }}$ & $\stackrel{0}{\circ}$ & $\begin{array}{l}\bar{N} \\
0 \\
1\end{array}$ & $\tilde{O}$ & $\begin{array}{l}\text { : } \\
0_{0}^{\prime}\end{array}$ & & $\begin{array}{l}n \\
0 \\
0 \\
i\end{array}$ & $o_{0}^{m}$ & & $\stackrel{\stackrel{n}{N}}{\sim}$ & & & $\overline{0}$ & & & & & & & & \\
\hline & & & & & & & & & & & & & & & & & & & & & & & & \\
\hline
\end{tabular}

